\title{
On the standard model group in F-theory
}

\author{
Kang-Sin Choi ${ }^{\mathrm{a}}$ \\ Scranton Honors Program, Ewha Womans University, Seoul 120-750, Korea
}

Received: 27 March 2014 / Accepted: 10 June 2014 / Published online: 24 June 2014

(C) The Author(s) 2014. This article is published with open access at Springerlink.com

\begin{abstract}
We analyze the standard model gauge group $S U(3) \times S U(2) \times U(1)$ constructed in F-theory. The nonAbelian part $S U(3) \times S U(2)$ is described by a surface singularity of Kodaira type. Blow-up analysis shows that the nonAbelian part is distinguished from the naïve product of $S U$ (3) and $S U(2)$, but that it should be a rank three group along the chain of $E_{n}$ groups, because it has non-generic gauge symmetry enhancement structure responsible for desirable matter curves. The Abelian part $U(1)$ is constructed from a globally valid two-form with the desired gauge quantum numbers, using a similar method to the decomposition (factorization) method of the spectral cover. This technique makes use of an extra section in the elliptic fiber of the Calabi-Yau manifold, on which F-theory is compactified. Conventional gauge coupling unification of $S U(5)$ is achieved, without requiring a threshold correction from the flux along the hypercharge direction.
\end{abstract}

\section{Introduction}

It is well-known wisdom that the gauge group $S U(3) \times$ $S U(2) \times U(1)$ of the standard model $(\mathrm{SM})$ is a far from arbitrary collection of simple and Abelian factors, because the matter fields transforming under the SM group have very particular charge assignments. Grand unified theory (GUT) $[1,2]$ suggests that it is best understood by embedding the group in a series of the exceptional groups $E_{n}$, including $S U(5) \equiv E_{4}, S O(10) \equiv E_{5}$, and $E_{6}$ [3]. In this sense the SM group may be expressed as the unique, maximal $E_{3} \times U(1)$ subgroup of $E_{4}$. These $E_{n}$ groups naturally occur in heterotic strings and F-theory [4-18].

In this work, we analyze the singularities and the twoforms describing the $\mathrm{SM}$ gauge group $S U(3) \times S U(2) \times U(1)$

\footnotetext{
a e-mail: kangsin@ewha.ac.kr
}

in F-theory. The non-Abelian part can be described by conventional Kodaira-type surface singularities in the elliptic fiber in the Calabi-Yau manifold on which F-theory is compactified [5,6,19-22]. Besides the sagacity that the GUT structure suggests, also in F-theory the desired light matter fields of the SM-not only the $(\mathbf{3}, \mathbf{2})$ representation but also $(\overline{\mathbf{3}}, \mathbf{1})$ and $(\mathbf{1}, \mathbf{2})$ - emerge on so-called matter curves [8$11,23]$, only if the $S U(3) \times S U(2)$ is realized as $E_{3}$ as above. This emergence is because essentially the matter fields can only arise by branching the gauge multiplets in the adjoint representations of (local) unified groups. With the clue of various gauge symmetry enhancement directions, we can obtain the desired singularities for $S U(3) \times S U(2)$ by deforming the singularity of $S U(5)[24,25]$. This subject will be reviewed and further analyzed by resolution of the corresponding singularity in Sect. 2. This analysis also provides additional evidence related to the shape of the $S U(3) \times S U(2)$ singularity.

In constructing the gauge theory, the real problem has been the construction of an Abelian $U(1)$ group that is not obtained from a Kodaira-type singularity. We obtain the Abelian gauge field by expanding the three-form tensor of M/F-theory along a two-cycle à la Kaluza-Klein reduction. For the elements of Cartan subalgebra of non-Abelian groups, we can automatically obtain the corresponding cycles by blowing up the singularity. On the other hand it is quite difficult to find such a $U(1)$ cycle which is globally valid and has an intersection structure giving the desired charges of the matter fields. Again, one hint can be embedding all the groups in a unified group, which would provide a two-form for the Abelian group in a similar fashion to the non-Abelian group. The socalled $U(1)$-restricted model was the first successful model to describe such a global $U(1)$ cycle for $S U(5) \times U(1)$ by embedding the $U(1)$ group into $S U(2)$ [26-30]. However, this process heavily depends on a clever choice of ansatz unique to $S U(5)$, and extending this technique to the general gauge group is not straightforward. Also there has been an indirect derivation of a globally valid $U(1)$ from the spectral 
cover [31] via heterotic-F-theory duality [32], which gives a globally valid two-form in the so-called stable degeneration limit. This derivation is useful in the case admitting the duality.

Recently, Mayrhofer et al. [33] and Esole and Yau [34] introduced a 'multiple section' method to introduce the $U(1)$, essentially by finding a cousin of the element from the Cartan subalgebra from a certain unified group (see also $[28,29,32,35])$. Such a two-form naturally arose from a more careful comparison between the elliptic curve and the spectral cover. In this paper, particularly in Sect. 3, we employ this method to obtain the $U(1)$ gauge fields along the desired directions in the group space like hypercharges. As a byproduct, this method provides another proof of the expression of the singularity $S U(3) \times S U(2)$ in relation to the spectral cover. We employed this method because it can be extended to the cases with any number of $U(1)$ sections. Also, although the exceptional divisors obtained by this method may not be directly embedded into a larger group such as $E_{8}$, the intersection relation can be embedded into and traced from such the group. Another interesting direction is to determine non-trivial sections of higher rank Mordell-Weil groups by embedding the elliptic fiber in a more general space $[24,36-$ 40].

At the moment, there are no clues whether we have the $\mathrm{SM}$ at the unification or the string/F-theory scale without an intermediate GUT. Besides such an a priori reason, the direct construction of the SM also has the following practical merits. First, we do not need to turn on a flux in the hypercharge direction, which gives rise to a threshold correction to the corresponding gauge coupling, ruining the coupling unification relation. Second, any sector related to electroweak symmetry breaking is better understood if we have the unbroken group as the SM group, since some fields as footprints of GUT have non-trivial coupling to the Higgs sector [41-45]. Even if the SM is realized at the unification scale, we still have footprints of GUT, such as the number of generations, since the string theory itself makes use of the unification relation $[24,25,46]$. These two features have no analogy in heterotic string theory, since in F-theory we have a two-step construction of gauge theory: constructing smaller groups than $E_{8}$ and further symmetry breaking by $G$-flux. The value of the flux controls the number of generations obeying a certain unification relation, while the actual gauge symmetry is the smaller SM group. Also there are several remaining technical issues. The hypercharge flux is difficult to construct in practice due to topological constraints; for example, when the base of the elliptic fibration has the $\mathbb{P}^{1}$ fiberation structure, thereby admitting heterotic duality. A related problem is that the three-generation relation of quarks and leptons from GUT may be broken if the hypercharge flux is induced on the matter curves of them.

\section{Non-Abelian factor $S U(3) \times S U(2)$}

We first construct a singularity for the non-Abelian algebra $S U(3) \times S U(2)$ of the SM. At the algebraic level, it is a mere direct product of the simple algebras $S U(3)$ and $S U(2) .{ }^{1}$ However, the subsequent analysis on the structure of the singularity by blow-ups reveals that the whole singularity describing $S U(3) \times S U(2)$ algebra should not be an arbitrary collection of those of $S U(3)$ and $S U(2)$ but it should be the singularity of an algebra along the $E_{n}$ series.

\subsection{Description by singularity}

We consider F-theory compactified on a Calabi-Yau fourfold $Y$ that is an elliptic fiberation over a three-base $B$ with a section. The elliptic fiber is given as a hypersurface by an equation in the 'Tate form'

$$
P \equiv-y^{2}+x^{3}+\mathrm{a}_{1} x y z+\mathrm{a}_{2} x^{2} z^{2}+\mathrm{a}_{3} y+\mathrm{a}_{4} x z^{4}+\mathrm{a}_{6} z^{6}=0
$$

in $\mathbb{P}_{[2,3,1]}^{2}$, with homogeneous coordinates $(x, y, z)$ with their respective weights indicated as subscripts. If we assume that all the parameters are appropriate holomorphic sections over $B$. Equation (1) can be regarded as the definition for our Calabi-Yau fourfold $Y$ as a hypersurface in the $\mathbb{P}^{2}$ fibration over $B$.

We construct the three-base $B$ such that there exists a divisor $W$ with a global holomorphic section $w$ serving as a normal coordinate to $W$, or admitting a definition

$W: w=0$,

up to change of the coordinate [21]. For example, requiring a $\mathbb{P}^{1}$ fiber in $B$ always admits this condition, if we take $w$ to be an affine coordinate of the $\mathbb{P}^{1}$ fiber. Then tuning the parameters $\mathrm{a}_{i}$ in this $w$ gives rise to a singularity related to a gauge symmetry on the worldvolume $W \times \mathbb{R}^{4}$. For instance, an $S U$ (5) singularity, also known as split $\mathrm{I}_{5}$, is obtained from the table by Kodaira [22]

$\mathrm{a}_{1}=b_{5}, \mathrm{a}_{2}=b_{4} w, \mathrm{a}_{3}=b_{2} w^{2}, \mathrm{a}_{4}=b_{2} w^{3}, \mathrm{a}_{6}=b_{0} w^{5}$,

up to higher order terms in $w$. The $W$ is the discriminant locus, since it is also given by vanishing locus of the discriminant of (1) [22],

$\Delta=b_{5}^{4}\left(b_{0} b_{5}^{2}-b_{2} b_{3} b_{5}+b_{3}^{2} b_{4}\right) w^{5}+O\left(w^{6}\right)$.

F-theory states that we have a gauge theory of $S U(5)$ supported on $W$, which we will analyze below [4-6,22].

${ }^{1}$ In this paper, we do not need to distinguish between the direct product of groups and the direct sum of algebras, following the conventional descriptions. 
Table 1 Parameters of gauge symmetry enhancements

\begin{tabular}{llll}
\hline Name & Parameter & Representation & Symm. enhancement \\
\hline$P_{(3,2)}$ & $b_{6}$ & $\mathbf{( 3 , 2 )}$ & $S U(5)$ \\
$P_{(\overline{3}, 1)}$ & $b_{3}^{2} b_{4} b_{5}-b_{2} b_{3} b_{5}^{2}+b_{0} b_{5}^{3}-b_{3}^{3} b_{6}$ & $(\overline{\mathbf{3}}, \mathbf{1})$ & $S U(4) \times S U(2)$ \\
$P_{(1,2)}$ & $b_{3}^{2} b_{4}-b_{2} b_{3} b_{5}+b_{0} b_{5}^{2}+b_{2}^{2} b_{6}-4 b_{0} b_{4} b_{6}$ & $(\mathbf{1}, \mathbf{2})$ & $S U(3) \times S U(3)$ \\
\hline
\end{tabular}

Deforming the singularity by adding lower order terms in $w$ to $\mathrm{a}_{i} \mathrm{~s}$, we can produce a less severe singularity describing a subgroup. It is claimed [24,25] that the singularity for the $S U(3) \times S U(2)$ is given by

$$
\begin{aligned}
& \mathrm{a}_{1}=b_{5}+O(w), \\
& \mathrm{a}_{2}=b_{4} w+O\left(w^{2}\right), \\
& \mathrm{a}_{3}=b_{3}\left(b_{6}+w\right) w+O\left(w^{3}\right), \\
& \mathrm{a}_{4}=b_{2}\left(b_{6}+w\right) w^{2}+O\left(w^{4}\right), \\
& \mathrm{a}_{6}=b_{0}\left(b_{6}+w\right)^{2} w^{3}+O\left(w^{6}\right) .
\end{aligned}
$$

The discriminant takes the form

$\Delta=b_{5}^{3} P_{(3,2)}^{2} P_{(\overline{3}, 1)} w^{3}+P_{(3,2)} P_{30} w^{4}+O\left(w^{5}\right)$,

where the parameters are displayed in Table 1 and $P_{30}$ is a quite lengthy, non-factorizable polynomial in $b_{i}$, e.g. containing a term $2 b_{3}^{2} b_{4} b_{5}^{4}$.

We have a split $\mathrm{I}_{3}$ singularity for $S U(3)$ located at $(x, y, w)=(0,0,0)$, which has orders ord $\left(\mathrm{a}_{1}, \mathrm{a}_{2}, \mathrm{a}_{3}, \mathrm{a}_{4}, \mathrm{a}_{6}\right.$, $\Delta)=(0,0,1,2,3,3)$ in $w[21,22]$. As a consequence, we can state that the $S U$ (3) gauge theory is supported on the $W$ in (2) [4-6].

We have local gauge symmetry enhancements along the following curves in $B$. In Table 1 , each equation $P_{f}=0$ defines a codimension-one curve of the $S U$ (3) surface $e_{0}=0$ and/or the $S U(2)$ surface $w^{\prime}=0$. Since we can infer that there are light matter fields $f$ localized on the $P_{f}=0$, we call this curve the matter curve [23]. Along $P_{(3,2)}=0$ the discriminant becomes degree five and the gauge symmetry is enhanced to $S U(5)$. $(\mathbf{3}, \mathbf{2})$ is regarded as an off-diagonal component of the adjoint $\mathbf{2 4}$ under the breaking $S U(5) \rightarrow$ $S U(3) \times S U(2)$. Likewise, along $P_{(\overline{3}, 1)}=0$, while $(\overline{\mathbf{3}}, \mathbf{1})$ is that of $\mathbf{1 5}$ under $S U(4) \rightarrow S U(3)$. The former symmetry enhancement shows that the actual group from the parameters (4)-(8) is larger than $S U$ (3). It is because the parameters are specially tuned up to ord $\left(\mathrm{a}_{1}, \mathrm{a}_{2}, \mathrm{a}_{3}, \mathrm{a}_{4}, \mathrm{a}_{6}\right)=(0,1,2,3,5)$ in $w$, as a deformation of the $\mathrm{I}_{5}$ singularity in (3). This structure is due to the identification of $S U(3) \times S U(2)$ as the $E_{3}$ group, as alluded to in the Sect. 1.

To see the other part, we change the reference as

$w^{\prime} \equiv w+b_{6}$

defining a new divisor $W^{\prime}: w^{\prime}=0$ of $B$. The parameters become
$\mathrm{a}_{1}=b_{5}+O\left(w^{\prime}\right)$,

$\mathrm{a}_{2}=b_{4}\left(w^{\prime}-b_{6}\right)+O\left(w^{\prime 2}\right)$,

$\mathrm{a}_{3}=b_{3}\left(w^{\prime}-b_{6}\right) w^{\prime}+O\left(w^{\prime 3}\right)$,

$\mathrm{a}_{4}=b_{2}\left(w^{\prime}-b_{6}\right)^{2} w^{\prime}+O\left(w^{\prime 4}\right)$,

$\mathrm{a}_{6}=b_{0}\left(w^{\prime}-b_{6}\right)^{3} w^{\prime 2}+O\left(w^{\prime 6}\right)$.

The discriminant has the form

$$
\begin{aligned}
\Delta= & \left(b_{5}^{2}-4 b_{4} b_{6}\right)^{2} P_{(3,2)}^{3} P_{(1,2)} w^{\prime 2} \\
& +P_{(3,2)}^{2} P_{30}^{\prime} w^{\prime 3}+P_{(3,2)} P_{41} w^{\prime 4}+O\left(w^{\prime 5}\right),
\end{aligned}
$$

where the parameters are shown in Table 1 , and $P_{30}^{\prime}, P_{41}$ are non-factorizable polynomials containing, respectively, $3 b_{3}^{2} b_{4} b_{5}^{4},-3 b_{3}^{2} b_{4} b_{5}^{5} b_{6}$. From the observation that we have the orders ord $\left(a_{1}, a_{2}, a_{3}, a_{4}, a_{6}, \Delta\right)=(0,0,1,1,2,2)$ in $w^{\prime}$ we see at $\left(x, y, w^{\prime}\right)=(0,0,0)$ that there is the Kodaira singularity, split $\mathrm{I}_{2}$ for $S U(2)$. Again we have the $S U(2)$ gauge theory localized on the locus $W^{\prime}$. The parameter $w^{\prime}$ is distinguished from $w$ by the relation (10) via the parameter $b_{6}$. We see the parameter $P_{(3,2)}=b_{6}$ again, since $(\mathbf{3}, \mathbf{2})$ is also charged under $S U(2)$. Along its vanishing locus, the gauge symmetry is enhanced to $S U(5)$. In this limit we do not distinguish between $w$ and $w^{\prime}$. Also $P_{(1,2)}=0$ enhances the symmetry $S U(2) \rightarrow S U(3)$. It is clear that our singularity describes the maximal semisimple algebra $S U(3) \times S U(2)$ embedded in $S U(5)$.

\subsection{Resolution}

We resolve the $S U(3) \times S U(2)$ singularity following the Tate algorithm [22,30,47]. This resolution reveals the structure of the singularity for the gauge group and its matter fields, which cannot be captured at the level of algebra, in terms of intersection theory. We rewrite the fiber equation, now neglecting higher order terms in $w$,

$$
\begin{aligned}
P= & -y^{2}+x^{3}+b_{5} x y z+b_{4} w x^{2} z^{2}+b_{3}\left(b_{6}+w\right) w y z^{3} \\
& +b_{2}\left(b_{6}+w\right) w^{2} x z^{4}+b_{0}\left(b_{6}+w\right)^{2} w^{3} z^{6} .
\end{aligned}
$$

First we resolve the $\mathrm{I}_{3}$ part of the singularity located at $(x, y, w)=(0,0,0)$. We transform the coordinates as

$(x, y, w)=\left(x_{1} e_{1}, y_{1} e_{1}, w_{1} e_{1}\right)$

and forbid the simultaneous vanishing $x_{1}=y_{1}=w_{1}=0$. We have introduced one more coordinate $e_{1}$ that is to be reduced. By introducing a scaling, 
Table 2 Scaling relations from the definition of the exceptional divisors $e_{1}, e_{2}$, and $e . w^{\prime}$ is a redundant coordinate according to the relation (19)

\begin{tabular}{llllrrrrr}
\hline & $x$ & $y$ & $z$ & $e_{1}$ & $e_{2}$ & $e$ & $e_{0}$ & $w^{\prime}$ \\
\hline$Z$ & 2 & 3 & 1 & 0 & 0 & 0 & 0 & 0 \\
$E_{1}$ & 1 & 1 & 0 & -1 & 0 & 0 & 1 & 0 \\
$E_{2}$ & 1 & 2 & 0 & 0 & -1 & 0 & 1 & 0 \\
$E$ & 1 & 1 & 0 & 0 & 0 & -1 & 0 & 1 \\
\hline
\end{tabular}

$\left(x_{1}, y_{1}, e_{1}, w_{1}\right) \rightarrow\left(\lambda x, \lambda y, \lambda^{-1} e_{1}, \lambda w_{1}\right)$,

which is well defined with the above excluded point, the dimension of the manifold remains the same. All of the scalings used here are shown in Table 2 . Then the original singular point is only accessed by $e_{1}=0$, which is the blown-up $\mathbb{P}^{1}$. However, the resulting manifold is not smooth yet: The lowest order terms in $e_{1}$ of $P$ have a common factor $y_{1}$, so the point $\left(e_{1}, y_{1}\right)=(0,0)$ is still singular. Therefore we blow up again

$\left(e_{1}, y_{1}\right)=\left(e_{1}^{\prime} e_{2}, y_{2} e_{2}\right)$,

or equivalently $(x, y, w)=\left(x_{1} e_{1}^{\prime} e_{2}, y_{2} e_{1}^{\prime} e_{2}^{2}, w_{1} e_{1}^{\prime} e_{2}\right)$, and we remove $e_{1}^{\prime}=y_{2}=0$. It should also be supplemented by another scaling as displayed in Table 2. Then the lowest order terms now in $e_{2}$ have no common factor and the resolution procedure terminates. From now on we drop the subscripts in $x_{1}, y_{1}, y_{2}, w_{1}$, and the prime in $e_{1}^{\prime}$, without danger of confusion. The resulting polynomial is

$$
\begin{aligned}
\tilde{P}= & e_{1}^{2} e_{2}^{3}\left[x^{3} e_{1}-y^{2} e_{2}+b_{5} x y z+b_{4} e_{1} w x^{2} z^{2}\right. \\
& +b_{3}\left(b_{6}+e_{1} e_{2} w\right) w y z^{3} \\
& +b_{2}\left(b_{6}+e_{1} e_{2} w\right) e_{1} w^{2} x z^{4} \\
& \left.+b_{0}\left(b_{6}+e_{1} e_{2} w\right)^{2} e_{1} w^{3} z^{6}\right] .
\end{aligned}
$$

Next, we move to the $S U(2)$ part, by using the relation (10), now possessing the form

$b_{6}+e_{1} e_{2} w \equiv w^{\prime}$.

(Here $w$ refers to $w_{1}$ in the above (14).) We obtain

$$
\begin{aligned}
\tilde{P}^{\prime}= & x^{3} e_{1}^{3} e_{2}^{3}-y^{2} e_{1}^{2} e_{2}^{4}+b_{5} e_{1}^{2} e_{2}^{3} x y z \\
& +b_{4} e_{1}^{2} e_{2}^{2}\left(w^{\prime}-b_{6}\right) x^{2} z^{2}+b_{3} w^{\prime}\left(w^{\prime}-b_{6}\right) e_{1} e_{2}^{2} y z^{3} \\
& +b_{2} w^{\prime}\left(w^{\prime}-b_{6}\right)^{2} e_{1} e_{2} x z^{4}+b_{0} w^{\prime 2}\left(w^{\prime}-b_{6}\right)^{3} z^{6} .
\end{aligned}
$$

As given above, this expression describes the $\mathrm{I}_{2}$ singularity at $\left(x, y, w^{\prime}\right)=(0,0,0)$. We want to blow up this point by introducing another coordinate $e$ such that

$$
\begin{aligned}
& \left(x, y, w^{\prime}\right) \rightarrow\left(x e, y e, w^{\prime} e\right), \\
& \hat{P}^{\prime}=e^{2}\left[x^{3} e_{1}^{3} e_{2}^{3} e-y^{2} e_{1}^{2} e_{2}^{4}+b_{5} e_{1}^{2} e_{2}^{3} x y z\right. \\
& \quad+b_{4} e_{1}^{2} e_{2}^{2}\left(w^{\prime} e-b_{6}\right) x^{2} z^{2}+b_{3} w^{\prime}\left(w^{\prime} e-b_{6}\right) e_{1} e_{2}^{2} y z^{3} \\
& \left.\quad+b_{2} w^{\prime}\left(w^{\prime} e-b_{6}\right)^{2} e_{1} e_{2} x z^{4}+b_{0} w^{\prime 2}\left(w^{\prime} e-b_{6}\right)^{3} z^{6}\right] .
\end{aligned}
$$

This is the standard resolution of the $I_{2}$ singularity, found in e.g. Ref. [33]. In these primed coordinates, we would seemingly have more singularities such as $\left(e_{2}, w^{\prime}\right)=(0,0)$. Shortly, as we will see, it will turn out that we do not have more singularities. We come back to the original coordinates, now by the modified relation

$w^{\prime} e-b_{6}=e_{1} e_{2} e_{0}$.

The coordinate $w$ is renamed to $e_{0}$ for later convenience. Then the equation becomes

$$
\begin{aligned}
\hat{P}= & e_{1}^{2} e_{2}^{3}\left[x^{3} e^{3} e_{1}-y^{2} e^{2} e_{2}+b_{5} e^{2} x y z+b_{4} e_{0} e_{1} e^{2} x^{2} z^{2}\right. \\
& +b_{3}\left(e_{0} e_{1} e_{2}+b_{6}\right) e_{0} e y z^{3}+b_{2}\left(e_{0} e_{1} e_{2}+b_{6}\right) e_{0}^{2} e_{1} e x z^{4} \\
& \left.+b_{0}\left(e_{0} e_{1} e_{2}+b_{6}\right)^{2} e_{0}^{3} e_{1} z^{6}\right] .
\end{aligned}
$$

The two resolution procedures should commute. Indeed we see they do, since we can write the overall result as

$\left(x, y, w, w^{\prime}\right) \rightarrow\left(x e e_{1} e_{2}, y e e_{1} e_{2}^{2}, e_{0} e_{1} e_{2}, w^{\prime} e\right)$,

with the scaling relations given in Table 2. The two resolutions are not independent due to the constraint (19).

A combination of two scalings always gives a new scaling in a different guise, and we see that it has the structure of an ideal. So we introduce the Stanley-Reisner (SR) ideal containing such data, generated by

$$
\begin{aligned}
& \left\{x y z, x y e_{0}, y e_{1}, x e_{0} e_{2}, y z e, x z e_{2}, z e_{1} e_{2}, x y w^{\prime}\right\} \\
& \cup\left\{\left(z e_{1}, z e\right) \text { xor } x e_{0}\right\} \cup\left\{z e_{2} \text { xor } y e_{0}\right\} \cup\left\{e_{1} w^{\prime} \text { xor } e e_{0}\right\} \\
& \cup\left\{e_{2} w^{\prime} \text { xor yee } e_{0}\right\} \cup\left\{z e e_{2} \text { xor } e_{0} w^{\prime}\right\} \\
& \cup\left\{x e_{2} w^{\prime} \text { xor }\left(e e_{0}, e_{1} e\right)\right\} \cup\left\{x e_{1} w^{\prime} \text { xor } e_{2} e\right\}
\end{aligned}
$$

Here, using xor in the parentheses (meaning exclusive or), we can choose one of the elements, corresponding to a particular triangulation of the toric diagram [48]. We must choose $z e_{1}$ and $z e_{2}$ to have four-dimensional Lorentz vector components for the Cartan subalgebras that will be related to $e_{1}$ and $e_{2}$, which we will explore below. We choose $e e_{0}, e_{2} w^{\prime}, e_{0} w^{\prime}, e_{1} e$, and $x e_{1} w^{\prime}$ here, some of which are generated by other ones. Therefore we finally have the SR ideal

$\left\{x y z, x y e_{0}, y e_{1}, x e_{0} e_{2}, z e_{1}, z e_{2}, z e, x y w^{\prime}, e_{0} e, e_{1} e, e_{0} w^{\prime}, e_{2} w^{\prime}\right\}$.

Every element indicates the condition that all of the parameters in it are not simultaneously zero. Such a guarantee of non-vanishing parameters will be used for simplifying the equation.

This completes the resolution and we are ready to define the smooth Calabi-Yau fourfold $\hat{Y}$ :

$\hat{P}=0$,

supplemented by the SR ideal (22). 


\subsection{Intersections}

We consider divisors of the smooth Calabi-Yau fourfold $\hat{Y}$ and calculate their intersections. The vanishing locus of each blow-up coordinate $e_{i}$ defines an exceptional divisor $E_{i}$. Explicitly we have

$$
\begin{aligned}
& E_{1}: e_{1}=0=-e_{2}+b_{5} x+b_{3} b_{6} e_{0}, \quad\left\{y e_{1}, z e_{1}, e e_{1}\right\} \\
& E_{2}: e_{2}=0=x^{3} e^{3} e_{1}+b_{5} e^{2} x y+b_{4} e_{0} e_{1} e^{2} x^{2} \\
& \quad+b_{3} b_{6} e e_{0} y+b_{2} b_{6} e e_{0}^{2} e_{1} x+b_{0} e_{0}^{3} e_{1} b_{6}^{2}, \quad\left\{z e_{2}\right\} \\
& \begin{aligned}
E_{0}: e_{0}= & 0=x^{3} e_{1}-y^{2} e_{2}+b_{5} x y z=0, \quad\left\{e_{0} e\right\}
\end{aligned} \\
& E: e=0=-y^{2} e_{2}^{4}+b_{5} e_{2}^{3} x y-b_{4} b_{6} e_{2}^{2} x^{2} \\
& \quad-b_{3} b_{6} w^{\prime} e_{2}^{2} y+b_{2} b_{6}^{2} e_{2} w^{\prime} x-b_{0} b_{6}^{3} w^{\prime 2}, \quad\left\{e e_{1}, z e\right\} \\
& W^{\prime}: w^{\prime}=0=x^{3} e_{1} e-y^{2}+b_{5} x y z-b_{4} b_{6} x^{2} z^{2}, \quad\left\{e_{0} w^{\prime}, e_{2} w^{\prime}\right\} .
\end{aligned}
$$

Here we simplified the relations by removing non-vanishing parameters using the SR ideal (22). The divisors $E_{1}, E_{2}$, and $E_{0}$ are the objects in the $S U(3)$ part, so we obtain them from $\hat{P} / e_{1}^{2} e_{2}^{3}$ after performing the proper transform given in (20). Likewise we obtain $E$ and $W^{\prime}$ of the $S U(2)$ from $\hat{P}^{\prime} / e^{2}$ in (18). To define $W^{\prime}$, we simply dropped the factor $e_{1}^{2} e_{2}^{2}$ in the equation $\left(\hat{P} / e^{2}\right)_{w^{\prime}=0}=0$, since the constraint (19) forbids either factor from vanishing. Using the constraint $e_{2}=-b_{6}$, we may change (26) into a fancier form, which will become important later.

We are mainly interested in the gauge quantum numbers that will be related to the intersection numbers between the following kind of curves. Let $\pi$ be the fiber map from $\hat{Y}$ to $B$, and $D_{a}, D_{b}$ be arbitrary divisors of the base intersecting transversally to $W$. In $\hat{Y}$, a complete intersection among $E_{i}, \pi^{*} D_{a}, \pi^{*} D_{b}$ gives a $\mathbb{P}^{1}$ curve. The intersection number of two such $\mathbb{P}^{1}$ curves from $E_{i}$ and $E_{j}$ is given by the number of common solutions to the equations $e_{i}=e_{j}=\hat{P} / e_{1}^{2} e_{2}^{3}=0$ :

$E_{1} \cdot E_{2}=1: \quad e_{1}=e_{2}=b_{5} x+b_{3} b_{6} e_{0}=0$,

$E_{1} \cdot E_{0}=1: \quad e_{1}=e_{0}=-e_{2}+b_{5} x=0$,

$E_{2} \cdot E_{0}=1: \quad e_{2}=e_{0}=e_{1}+b_{5} y=0, \quad\left\{x e_{0} e_{2}, e_{0} e, e_{2} e\right\}$,

where the dot product notation is understood. Each equation has one solution in $x, y$, and/or $e_{i}$, assuming that all $b_{i}$ are nonzero. This completes the $S U$ (3) root relations via the McKay correspondence to the intersection numbers corresponding to the negative of the Cartan matrix of the algebra. The above intersections can also be expressed as $[49,50]$

$$
\int_{\hat{Y}} E_{i} \wedge E_{j} \wedge \pi^{*} D_{a} \wedge \pi^{*} D_{b}=-A_{i j} \int_{B} W \wedge D_{a} \wedge D_{b},
$$

where $A_{i j}$ is the Cartan matrix of $S U(3)$. These exceptional divisors $E_{1}, E_{2}, E_{0}$ correspond to the roots in the extended $S U$ (3) algebra. They are disconnected to the rest of the divisors $E$ and $W^{\prime}$ of the $S U(2)$, since the constraint does not allow the simultaneous vanishing of $e_{i}$ and $e$, or of $e_{i}$ and $w^{\prime}$ for each $i=1,2,0$.

For $E$ and $W^{\prime}$, we have two solutions in $y / x$ to

$$
\begin{aligned}
& E \cdot W^{\prime}=2: \quad e=w^{\prime}=y^{2}-b_{5} x y \\
& +b_{4} b_{6} x^{2}=0, \quad\left\{e z, e_{0} e, e_{1} e, e_{2} w^{\prime}\right\}
\end{aligned}
$$

consistent with the extended Dynkin diagram of $S U(2)$. If there is only one solution, the discriminant of (32) becomes

$b_{5}^{2}-4 b_{4} b_{6}=0$,

which destroys the $O\left(w^{\prime 2}\right)$ term in the discriminant (12). For the valid existence of the $S U(2)$, we will assume that there is only one solution in what follows.

\subsection{Matter curves and symmetry enhancement}

In Sect. 2.1, we have studied gauge symmetry enhancements along various matter curves. Using the results of the previous section, we now calculate explicit gauge quantum numbers of the fields arising from the matter curves.

Matter curves for $(3,2)$ We first analyze the matter curve $P_{(3,2)}=0$ for $(\mathbf{3}, \mathbf{2})$. On this locus, there is local gauge symmetry enhancement to $S U(5)$.

The equations for exceptional divisors become further factorized and the resulting exceptional divisors degenerate,

$$
\begin{aligned}
& E_{1} \rightarrow E_{1 A}: \quad-e_{2}+b_{5} x=0=e_{1}, \\
& E_{2} \rightarrow E_{2 x} \cup E_{2 E} \cup E_{2 B}: \quad x e^{2}\left(x^{2} e e_{1}+b_{5} y+b_{4} e_{0} e_{1} x\right) \\
& \quad=0=e_{2}, \\
& E_{0} \rightarrow E_{0 C}: \quad x^{3} e_{1}-y^{2} e_{2}+b_{5} x y z=0=e_{0} \\
& E \rightarrow E_{2 E}: \quad e_{2}^{3} y\left(-y e_{2}+b_{5} x\right)=0=e .
\end{aligned}
$$

We renamed the divisors accordingly for obvious reasons. We may find a divisor $E_{2 E}$ by $e_{2}=e=0$ not only from $E_{2}$ but also from $E$. In particular, the previously disconnected parts $E_{2}$ and $E$ can communicate via this relation. Only this satisfies the modified constraint (19), which now reads $e=e_{2}$ using the SR elements $\left\{e_{0} e, e_{1} e, e_{2} w^{\prime}\right\}$. This entails only the possible divisor from $E$ to be $E_{2 E}$, although other ones such as $e=y=0$ are seemingly possible.

Consequently, the only non-trivial intersections are

$$
\begin{array}{ll}
E_{0 C} \cdot E_{1 A}=1: & e_{0}=e_{1}=-e_{2}+b_{5} x=0, \\
E_{1 A} \cdot E_{2 x}=1: & e_{2}=e_{1}=x=0, \\
E_{2 E} \cdot E_{2 x}=1: & e_{2}=e=x=0, \\
E_{2 E} \cdot E_{2 B}=1: & e_{2}=e=b_{5} y+b_{4} x=0, \quad\left\{e_{0} e\right\}, \\
E_{2 B} \cdot E_{0 C}=1: & e_{2}=e_{0}=e_{1}+b_{5} y=0 .
\end{array}
$$


Table $3 S U(3) \times S U(2)$ weights of the exceptional divisors in the Dynkin basis

\begin{tabular}{ll}
\hline Divisor & Weight \\
\hline$E_{1 A}$ & {$[-2,1 ; 0]$} \\
$E_{2 x}$ & {$[1,-1 ; 1]$} \\
$E_{2 E}$ & {$[0,0 ;-2]$} \\
$E_{2 B}$ & {$[0,-1 ; 1]$} \\
$E_{0 C}$ & {$[1,1 ; 0]$} \\
\hline
\end{tabular}

Table 4 The components of the matter representations $(3,2)$, at the matter curve $P_{(3,2)}=0$

\begin{tabular}{ll}
\hline Divisor & Weight \\
\hline$E_{2 B}+E_{0 C}$ & {$[1,0 ; 1]$} \\
$E_{2 B}+E_{0 C}+E_{2 E}$ & {$[1,0 ;-1]$} \\
$E_{2 B}+E_{0 C}+E_{1 A}$ & {$[-1,1 ; 1]$} \\
$E_{2 B}+E_{0 C}+E_{1 A}+E_{2 E}$ & {$[-1,1 ;-1]$} \\
$E_{2 B}$ & {$[0,-1 ; 1]$} \\
$E_{2 B}+E_{2 E}$ & {$[0,-1 ;-1]$} \\
\hline
\end{tabular}

The other intersection numbers are zero. For instance, there is no intersection between $E_{2 x}$ and $E_{0 C}$, since $e_{0}=e_{2}=x=0$ is forbidden by the SR element $x e_{0} e_{2}$. In particular there is no intersection between $E_{2 x}$ and $E_{2 B}$, since the required equations $e_{2}=x=y=0$ are forbidden by the SR elements $\left\{x y w^{\prime}, e_{2} w^{\prime}\right\}$.

These relations give rise to the extended Lie algebra of the locally enhanced gauge symmetry $S U(5)$. However, the unbroken gauge symmetry on $\hat{Y}$ still globally remains as $S U(3) \times S U(2)$. Although the divisor $E$ had no intersections with $E_{2}$ or $E_{0}$ before the symmetry enhancement, now the degenerate daughters of the $E$ do have nonzero intersections. This fact can be tracked to general factors $e$ appearing in the divisors, resulting from the resolution of the $\mathrm{I}_{2}$ part, which cannot be separately done with respect to the $\mathrm{I}_{3}$ part.

Also we can calculate the $S U(3) \times S U(2)$ weights of the divisors in (34)-(37) in the Dynkin basis, $\left[E_{i} \cdot E_{1}, E_{i} \cdot E_{2} ; E_{i}\right.$. $E$ ], as shown in Table 3. On the matter curve $P_{(3,2)}=0$, we have a local gauge symmetry enhancement, which explains the emergence of a light field with a quantum number $(\mathbf{3}, \mathbf{2})$. The weights of its six components are displayed in Table 4 . As expected, two roots of $S U(3)$ are played by $E_{1 A}$ and $E_{2 x}+E_{2 E}+E_{2 B}$, and the root of $S U(2)$ is $E_{2 E}$. One can easily see that these highest weight representations are the only ones whose divisor components are all effective.

Matter curves for $(\overline{\mathbf{3}}, \mathbf{1})$ There are other gauge symmetry enhancement directions, according to Table 1 . We have an $S U(3) \rightarrow S U(4)$ symmetry enhancement, yielding light matter $(\overline{\mathbf{3}}, \mathbf{1})$ at the matter curve $P_{(\overline{3}, 1)}=0$. For example solving this equation in $b_{6}$ and restoring appropriately $b_{6}$
Table 5 The components of the matter representations $(\overline{\mathbf{3}}, \mathbf{1})$ at the matter curve $P_{(\overline{3}, 1)}=0$, and $(\mathbf{1}, \mathbf{2})$ at those of the matter curve $P_{(1,2)}=0$

\begin{tabular}{llll}
\hline Divisor & Weight & Divisor & Weight \\
\hline$E_{0}+E_{1}+E_{2 F}$ & {$[-1,1 ; 0]$} & $E_{G}$ & {$[0,0 ; 1]$} \\
$E_{2 F}$ & {$[0,-1 ; 0]$} & $E_{G}+E$ & {$[0,0 ;-1]$} \\
$E_{1}+E_{2 F}$ & {$[-2,0 ; 0]$} & & \\
\hline
\end{tabular}

Table 6 Defining equation for the matter curves

\begin{tabular}{ll}
\hline Matter & Parameters \\
\hline$P_{X}$ & $a_{1}$ \\
$P_{q_{\circ}}$ & $d_{5}$ \\
$P_{u_{\circ}^{c}}$ & $a_{1}^{3} d_{2}+a_{0} a_{1}^{2} d_{3}+a_{0}^{2} a_{1} d_{4}+a_{0}^{3} d_{5}$ \\
$P_{d_{\circ}^{c}}$ & $a_{0} a_{1} d_{2} d_{3} d_{4}+a_{0}^{2} d_{3}^{2} d_{4}+a_{1}^{2} d_{0} d_{4}^{2}-a_{0} a_{1} d_{2}^{2} d_{5}$ \\
& $\quad-a_{0}^{2} d_{2} d_{3} d_{5}+2 a_{0} a_{1} d_{0} d_{4} d_{5}+a_{0}^{2} d_{0} d_{5}$ \\
$P_{l_{\circ}}$ & $a_{1}^{5} d_{5} d_{0}^{2}-a_{1}^{3} d_{5} a_{0}^{2} d_{2} d_{0}-2 a_{1} d_{5} a_{0}^{4} d_{0} d_{4}-3 a_{1}^{2} d_{5} a_{0}^{3} d_{0} d_{3}$ \\
& $+a_{0}^{3} d_{0} a_{1}^{2} d_{4}^{2}+a_{0}^{5} d_{0} d_{5}^{2}+a_{0}^{4} d_{2} a_{1} d_{4} d_{3}-a_{0}^{5} d_{2} d_{5} d_{3}$ \\
& $+a_{1}^{3} d_{0} d_{4} a_{0}^{2} d_{3}+a_{0} a_{1}^{4} d_{0} d_{4} d_{2}+a_{0}^{5} d_{4} d_{3}^{2}+a_{0}^{4} a_{1} d_{3}^{3}$ \\
& $+2 a_{0}^{3} a_{1}^{2} d_{3}^{2} d_{2}+a_{0}^{2} a_{1}^{3} d_{3} d_{2}^{2}$ \\
& $-2 d_{1} a_{1}^{4}+d_{2} a_{0} a_{1}^{3}-d_{3} a_{0}^{2} a_{1}^{2}+d_{4} a_{0}^{3} a_{1}^{2}-d_{5} a_{0}^{4}$ \\
$P_{e_{\circ}^{c}}$ &
\end{tabular}

again, we recover a degeneration of $E_{2}$,

$$
\begin{aligned}
E_{2} & \rightarrow E_{2 D} \cup E_{2 F}: e_{2}=0 \\
= & \left(b_{3} b_{6} e_{0}+b_{5} e x\right)\left[b_{3} b_{5} b_{6} e_{0}^{2} e_{1}\right. \\
& \left.+b_{5}\left(b_{2} b_{3}-b_{0} b_{5}\right) e e_{0} e_{1} x+b_{3}^{2} e\left(b_{5} y+e e_{1} x^{2}\right)\right]
\end{aligned}
$$

dropping $b_{3}^{-2} b_{5}^{-1} b_{0} . E_{0}$ and $E_{1}$ are essentially unmodified.

We have $E_{0} \cdot E_{1}=1$ as before and in addition we have intersections of new divisors,

$$
\begin{array}{ll}
E_{1} \cdot E_{2 D}=1: & e_{1}=e_{2}=b_{5} x+b_{3} b_{6} e_{0}=0, \\
E_{1} \cdot E_{2 F}=0: & e_{1}=e_{2}=b_{3}^{4} b_{5}=b_{5} x+b_{3} b_{6}=0, \\
\quad\left\{e_{1} e, e_{1} y\right\}, & \\
E_{0} \cdot E_{2 D}=0: & e_{0}=e_{2}=x=0, \quad\left\{x e_{0} e_{2}\right\}, \\
E_{0} \cdot E_{2 F}=1: & e_{0}=e_{2}=e_{1}+b_{5} y=0, \quad\left\{x e_{0} e_{2}\right\} .
\end{array}
$$

The representations are calculated in Table 5, where we must take the highest weight $[-1,1 ; 0]$, not $[0,1 ; 0]$.

Matter curves for $(\mathbf{1}, \mathbf{2})$ Another symmetry enhancement direction is $P_{(1,2)}=0$, shown in Table 6 . We use the equation for the divisor $E$, after applying the constraint in (19), which now reads $e_{2}=-b_{6}$,

$$
E: e=0=b_{6} y^{2}+b_{5} x y+b_{4} x^{2}+b_{3} w^{\prime} y+b_{2} w^{\prime} x+b_{0} w^{\prime 2} \text {, }
$$


after dropping $-b_{6}^{3}$. The divisor $E$ in (48) may degenerate into two,

$E_{G} \cup E_{H}: e=0=\left(p w^{\prime}+q x+r y\right)\left(s w^{\prime}+u x+v y\right)$,

where the relations

$$
\begin{gathered}
b_{0}=p s, b_{2}=p u+q s, b_{3}=s r+p v, b_{4}=q u, \\
b_{5}=r u+q v, b_{6}=r v
\end{gathered}
$$

satisfy the condition $P_{(1,2)}=0$ in a highly non-trivial way. We can always solve the six parameters $p, q, r, s, u, v$ for as many $b_{i}$. In fact, setting $w^{\prime}=1$ makes (48) identical to the $S U$ (6) spectral cover equation, for which the condition for local factorization (49) is precisely the requirement for the existence of the $(\mathbf{1}, \mathbf{2}, \mathbf{2 0})$ representation of $S U(3) \times S U(2) \times$ $S U$ (6) $\subset E_{8}$ [13]. This fact is additional evidence that the divisor equation for $E$ in (48) should be obtained from $\hat{P}^{\prime}$, not from $\hat{P}$.

Accordingly, each $E_{G}$ or $E_{H}$ provides a representation for $(\mathbf{1}, \mathbf{2})$, shown in Table 5. We may check the intersection relations in the same way,

$E_{G} \cdot E_{H}=1: e=p w^{\prime}+q x+r y=s w^{\prime}+u x+v y=0$,

where we have a non-trivial solution to the last two equations in $x$ and $y$ if

$(u r-q v)^{2}=b_{5}^{2}-4 b_{4} b_{6} \neq 0$,

which we have already met in (33) and assumed to be nonvanishing. Since

$E_{G} \cdot E=E_{G} \cdot\left(E_{G}+E_{H}\right)=-2+1=-1$,

the same relation holds for $E_{H} \cdot E=-1$, by the exchange symmetry between $E_{G}$ and $E_{H}$. We see also that there is no distinction between the $E_{G}$ and $E_{H}$ group-theoretically, since $(\mathbf{1}, \mathbf{2})$ is a self-conjugate representation. But this situation may be subject to the Freed-Witten global anomaly.

\section{Abelian factor $U(\mathbf{1})$}

The work by Mayrhofer et al. [33], following that of Esole and Yau [34], introduced a systematic way to obtain a number of $U(1)$ gauge fields with desired gauge quantum numbers, whose methodology we follow here. The key idea is to introduce a new section rather than the zero section in the elliptic fiber, by deforming its complex structure reflecting some group structure. The resulting Calabi-Yau manifold becomes singular, and the small resolution of the singularity may give rise to the desired two-form. This situation is very similar and indeed related to the decomposition (factorization) of the spectral cover to which we can relate the group elements [32]. A non-trivially modified application to the $S U(3) \times S U(2)$ singularity shall give rise to the hypercharge $U(1)$.

\subsection{More global sections from factorization}

We require a number of one-forms $A_{1}$. These one-forms provide gauge fields for either the Cartan subalgebra of nonAbelian groups or just $U(1)$ groups. To realize these oneforms, we require harmonic two-forms $w_{2} \in H^{1,1}(\hat{Y})$ by which the M/F-theory three-form tensor is expanded as

$$
\begin{aligned}
C_{3} & =\sum A_{1} \wedge w_{2} \\
& =A_{1}^{e_{1}} \wedge w_{2}^{e_{1}}+A_{1}^{e_{2}} \wedge w_{2}^{e_{2}}+A_{1}^{e} \wedge w_{2}^{e}+A_{1}^{Y} \wedge w_{2}^{Y}+\cdots
\end{aligned}
$$

Here, $w_{2}^{e_{i}}$ are the dual two-forms to the divisors $E_{1}, E_{2}$ or $E$ of $\hat{Y}$ obtained from the blow ups described in the previous section.

We set two kinds of requirements for the $w_{2}$ : (i) Each $A_{1}$ should have the desired gauge quantum number, so that the Poincaré dual divisor of the paired $w_{2}$ in $\hat{Y}$ has appropriate intersections with other divisors, and (ii) every $A_{1}$ should be a seven-dimensional vector field, which restricts the index structure of the components of the paired $w_{2}$ [10]. For a resolved Kodaira singularity, the blown-up cycles $w_{2}^{e_{i}}$ automatically satisfy requirement (i), as seen from the intersection structure seen in Sect. 2.3. However, there is no Kodaira singularity for an Abelian symmetry, or the would-be-related $\mathrm{I}_{1}$ singularity is actually smooth. Therefore, we cannot complete the resolution as in Sect. 2.2. We will see shortly that the desired two-form is obtained from another kind of singularity under a more special condition. The goal of this section is to find $w_{2}^{Y}$ harboring the hypercharge satisfying requirement (ii).

An important hint is the relation between the equation of an elliptic fiber and that of a spectral cover [31]. We give a rough sketch on how a two-form is obtained using the example of $S U(5)$, and fill in the details for the $S U(3) \times S U(2)$ case. We required a global section in the elliptic fiber, which is at $(x, y, z)=(1,1,0)$ in our case, and usually called the zero section $Z$. This section is lifted to a divisor of $Y$, given by the hypersurface equation $y^{2}-x^{3}=0$. Conversely on this divisor, the fiber equation becomes the spectral cover equation of the commutant group $S U(5)_{\perp}$ in $E_{8}$,

$\left.P\right|_{y^{2}=x^{3}}=z\left(b_{0} z^{5}+b_{2} t^{2} z^{3}+b_{3} t^{3} z^{2}+b_{4} t^{4} z+b_{5} t^{5}\right)$,

with a parametrization $t=y / x$. This fact shows that there can be another possible section other than $z=0$ in the fiber, if we have a further factorization with a linear polynomial in $z$. This is nothing but a factorization or decomposition condition in the spectral cover and we know how gauge theory information is encoded in it. The resulting manifold $Y$ has an 
extra singularity and we perform a small resolution (the resolution of a singularity of codimension higher than 1) to obtain a new divisor. We may hope that this new divisor provides a two-form with the desirable gauge quantum numbers from intersection structures with the existing resolution divisors. Strictly speaking, the relation should be used when we have a stable degeneration limit. However, later we will verify that without such a limit the new divisor does have the correct intersection structure.

Although the new section is easily found in the $S U(5)$ case, the conversion of the fiber equation to a spectral cover equation is highly non-trivial in the $S U(3) \times S U(2)$ case. We claim that the following divisor gives the desirable section for the hypercharge $U(1)$ :

$$
X \equiv e_{0} e_{1}^{2} e x^{3}-\left(e_{0} e_{1} e_{2}+b_{6}\right) y^{2}=e\left(e_{0} e_{1}^{2} x^{3}-w^{\prime} y^{2}\right)=0 .
$$

Since the exceptional divisors are not relevant to our discussion of the new divisor harboring $U(1)$, the essential fact here is the factor $e_{0}=w$ and $w^{\prime}$ in front of $x^{3}$ and $y^{2}$, respectively. It is convenient to define $t$ as

$x e_{1} t \equiv y, \quad\left\{e_{1} y, x y\right\}$,

serving as a well-behaved holomorphic coordinate. It is because the condition $t=0$ means $y=0$, and the converse also holds because the SR elements indicated in (55) forbid $x=0$ and $e_{1}=0$. So from now on we use $t=y e_{1}^{-1} x^{-1}$, by which (54) becomes

$e_{0} e x-\left(e_{0} e_{1} e_{2}+b_{6}\right) t^{2}$.

On this divisor $X=0$, the fiber polynomial $\hat{P}$ in (20) becomes

$$
\begin{aligned}
\left.\hat{P}\right|_{X=0}= & e_{0}^{-3} e_{1} e^{2} w^{\prime 2}\left(b_{0} e_{0}^{6} z^{6}+b_{2} t^{2} e_{0}^{4} z^{4}+b_{3} t^{3} e_{0}^{3} z^{3}\right. \\
& \left.+b_{4} t^{4} e_{0}^{2} z^{2}+b_{5} t^{5} e_{0} z+b_{6} t^{6}\right) .
\end{aligned}
$$

Unless $b_{6}=0, E_{0}$ has no intersection with $X$ so we do not care about the factor $e_{0}^{-3}$, otherwise there is a cancelation and no overall factor in $e_{0}$ remains. Besides the prefactor, the polynomial in $z$, in the parentheses, is nothing but the $S U$ (6) spectral cover equation. ${ }^{2}$ In the spectral cover description, the vanishing sum of five distinguished points on the elliptic fiber of the heterotic dual theory, for the unimodular group $S U$, should be translated to the absence of a $z^{5}$ term. This is consistent with our choice of the zero section, $z=0$.

In the spectral cover description, we have obtained a globally valid $U(1)$ by the 'decomposition' method [32]. Its first procedure is tuning of the parameters (4)-(8), as follows $[10,13,15,24,33,51]$ :

\footnotetext{
2 The relation between the equations for the elliptic fiber and for the spectral cover is discussed in Refs. $[13,33]$.
}

$b_{0}=a_{0} d_{1}, \quad b_{2}=a_{0} d_{2}+a_{1} d_{1}, \quad b_{3}=a_{0} d_{3}+a_{1} d_{2}$,

$b_{4}=a_{0} d_{4}+a_{1} d_{3}, \quad b_{5}=a_{0} d_{5}+a_{1} d_{4}, \quad b_{6}=a_{1} d_{5}$.

In other words,

$\mathrm{a}_{1}=\left(a_{0} d_{5}+a_{1} d_{4}\right)$,

$\mathrm{a}_{2}=\left(a_{0} d_{4}+a_{1} d_{3}\right) w$,

$\mathrm{a}_{3}=\left(a_{0} d_{3}+a_{1} d_{2}\right)\left(a_{1} d_{5}+w\right) w$,

$\mathrm{a}_{4}=\left(a_{0} d_{2}+a_{1} d_{1}\right)\left(a_{1} d_{5}+w\right) w^{2}$,

$\mathrm{a}_{6}=a_{0} d_{0}\left(a_{1} d_{5}+w\right)^{2} w^{3}$.

The absence of $\mathrm{a}_{5}$ or $b_{1}$ should be translated as a constraint,

$0=a_{0} d_{1}+a_{1} d_{0}$.

Then the Tate polynomial gets the factorized form

$$
\begin{aligned}
\left.\hat{P}\right|_{X=0}= & e_{0}^{-3} e_{1} e^{2} w^{\prime 2}\left(a_{0} e_{0} z+a_{1} t\right)\left(d_{0} e_{0}^{5} z^{5}+d_{1} e_{0}^{4} z^{4} t\right. \\
& \left.+d_{2} e_{0}^{3} z^{3} t^{2}+d_{3} e_{0}^{2} z^{2} t^{3}+d_{4} e_{0} z t^{4}+d_{5} t^{5}\right) \\
\equiv & e_{0}^{-3} e_{1} e^{2} w^{2} Y_{1} Y_{2} .
\end{aligned}
$$

This equation will correspond to the $S[U(1) \times U(5)]$ spectral cover equation on the heterotic side. However, to ensure that this $U(1)$ is global, we require another global section other than the zero section [24,33]. In our case, indeed we find that the new section is at $X=Y_{1}=0$. Since this section is related to the ' $U(1)$ part' $Y_{1}$ or the parameter $a_{1} / a_{0}$, we may expect that this section has the appropriate structure for the hypercharge.

The factorization structure (64) may be expressed in another way [33]:

$\hat{P}=X Q-Y_{1} Y_{2}=0$,

by introducing a polynomial $Q$, holomorphic in $z$ and $t$. This leads to a conifold singularity at

$X=Q=Y_{1}=Y_{2}=0$,

which is of higher codimension than 1 . We blow up $Y_{1}=$ $Q=0$ by introducing a $\mathbb{P}^{1}$ with homogeneous coordinates $\left(\lambda_{1}, \lambda_{2}\right)$ such that [34]

$Y_{1} \lambda_{2}=Q \lambda_{1}, \quad Y_{2} \lambda_{1}=X \lambda_{2}$.

The original singularity (66) gives unconstrained $\lambda_{1}$ and $\lambda_{2}$, which means that it is replaced by the $\mathbb{P}^{1}$. Away from the singularity, we recover the original equation (65) by solving $\lambda_{1}$ and $\lambda_{2}$. In effect, the equations in (67) have redefined the Calabi-Yau space, which we denote as $\hat{Y}$ again, by abusing the notation, as a hypersurface in the new ambient space including the $\mathbb{P}^{1}$. Then we obtain an extra section besides the zero section as the following divisor in $\hat{Y}$ :

$S: \lambda_{1}=0$,

which forces $\lambda_{2}$ to be nonzero and gives $Y_{1}=X=0$ from (67). The lesson from the spectral cover [32] tells us that 
$Y_{1}=0$ is related to the hypercharge $U(1)$ as a subset of the commutant $S[U(1) \times U(5)]$ to the SM group in $E_{8}$. The desired candidate for the hypercharge $(1,1)$-form is therefore the Poincaré dual to the threefold $S$ in $\hat{Y}$, up to some correction that we should consider below.

Next, we consider the requirement (ii) below Eq. (53), for $A_{1}$ being a Lorentz vector. A natural method is given in Ref. [33], which we follow here with similar notations. Such forms $w$ should satisfy the following constraints:

$$
\begin{aligned}
& \int_{\hat{Y}} w \wedge D_{a} \wedge D_{b} \wedge D_{c}=0, \\
& \int_{\hat{Y}} w \wedge Z \wedge D_{a} \wedge D_{b}=0,
\end{aligned}
$$

from which the two indices of $w$ have one leg on the elliptic fiber and the other leg on the base $B$. Here the divisors $D_{a}, D_{b}, D_{c}$ are arbitrary divisors in $B$ and we omitted the pullback by $\pi$. It is far from trivial for the Cartan subalgebra elements $E_{1}$ and $E_{2}$ to satisfy the relation (70), without choosing the SR ideal given in (21). For $w$ we find the desired linear combination

$w_{Y}=S-Z-\overline{\mathcal{K}}+a_{1}+\sum t_{i} E_{i}$,

where $\overline{\mathcal{K}}$ is the canonical class of the base $B, a_{1}$ is the divisor defined by $a_{1}=0$, and the coefficients $t_{i}$ will be determined later. This general process is called the Shioda map [52-55]. The condition (69) is satisfied using the relation

$$
\begin{aligned}
& \int_{\hat{Y}} S \wedge D_{a} \wedge D_{b} \wedge D_{c}=\int_{\hat{Y}} Z \wedge D_{a} \wedge D_{b} \wedge D_{c} \\
& \quad=\int_{B} D_{a} \wedge D_{b} \wedge D_{c},
\end{aligned}
$$

since both $S$ and $Z$ are global sections. The next condition (70) is satisfied by (71) thanks to the following. First, the intersection of $Z: z=0$ means $Y_{1}=a_{1} t$ among the defining equations of $S$. Using the fact that $x y z$ is an element of the SR ideal, the only non-triviality for the intersection of $S \cdot Z$ comes from

$$
\int_{\hat{Y}} S \wedge Z \wedge D_{a} \wedge D_{b}=\int_{B} a_{1} \wedge D_{a} \wedge D_{b} .
$$

Also the adjunction formula states that

$$
\int_{\hat{Y}} Z \wedge Z \wedge D_{a} \wedge D_{b}=-\int_{B} \overline{\mathcal{K}} \wedge D_{a} \wedge D_{b} .
$$

\subsection{More symmetry enhancement}

The procedure described in the previous subsection introduces two new things: one is the new section $S$ and the other is a further factorization of the matter curve. We calculate the intersection of the divisors with $S$ under various symmetry enhancement conditions on the matter curves. From this, we see that, although $S$ did not originate from the Cartan subalgebra of the $S U$ (5) singularity or Kodaira $\mathrm{I}_{5}$, it plays exactly the same role for the fourth generator of it, different from the existing generators $E_{1}, E_{2}, E$.

Matter curves for $(\mathbf{3}, \mathbf{2})$ Under the parametrization (59)-(63) the matter curve equation $P_{(3,2)}=0$ in (9) further factors as

$P_{(3,2)}=P_{q} P_{X}=0$,

with different intersection structures of the exceptional divisors for each factor shown in Table 6 . We can directly compute the intersections, omitting $\lambda_{1}=D_{a}=0$ :

$$
\begin{aligned}
& S \cdot E_{1 A, q}=0: \quad e_{1}=y=-e_{2}+a_{1} d_{4} x=d_{5}=0, \quad\left\{y e_{1}\right\} \\
& S \cdot E_{2 E, X}=1: \quad e_{2}=e=x=a_{1}=0, \quad\left\{x e_{0} e_{2}, e_{0} e, e_{1} e\right\} \\
& S \cdot E_{2 E, q}=1: \quad e_{2}=e=Y_{1}=d_{5}=0 \\
& S \cdot E_{2 B, X}=1: \quad e_{2}=e_{0}=e_{1}+a_{0} d_{5} y=a_{1}=0 \\
& \quad\left\{x e_{0} e_{2}, e_{0} e\right\}, \\
& S \cdot E_{2 B, q}=0: \quad e_{2}=X=Y_{1}=x^{2} e e_{1}+a_{1} d_{4} y \\
& \quad+\left(a_{1} d_{3}+a_{0} d_{4}\right) e_{0} e_{1} x=d_{5}=0, \\
& S \cdot E_{2 x, q}=0: \quad e_{2}=x=y=d_{5}=0, \quad\{x y\}
\end{aligned}
$$

In calculating the intersection of $Y_{1}=a_{0} e_{0}+a_{1} t$ with $x=0$ or $e_{1}=0$, the definition of $t$ is invalid, so we need to restore its original form $a_{0} e_{0} e_{1} x+a_{1} y$.

We should remember that, although we have local gauge symmetry enhancement, still on $\hat{Y}$ the gauge symmetry is $S U(3) \times S U(2) \times U(1)$, whose basis corresponds to $E_{1}, E_{2}, E$. Thus we have a definite product between those with $S$. We should have a definite intersection $S \cdot E_{1}=$ $S \cdot E_{1 A, q}=0$, so we should also have

$S \cdot E_{1 A, X}=0$.

As before, we have an invariant

$S \cdot E_{2}=S \cdot\left(E_{2 x}+E_{2 E}+E_{2 B}\right)=1$,

calculated from the matter curve $q$. Since $S \cdot E_{2}$ should be independent of the decomposition, we should have the same value for the $X$ and we have

$$
S \cdot E_{2 x, X}=-1 \text {. }
$$

Also indirectly we can obtain this value. From the definition of the extended root $E_{0}$, we recover the linear dependence 
relation $E_{0 C}+E_{1 A}+E_{2 x}+E_{2 E}+E_{2 B}=0$, fixing, for both $X$ and $q$,

$S \cdot E_{0 C}=-1$.

Matter curves for $(\overline{\mathbf{3}}, \mathbf{1})$ Now we go to the case of $(\overline{\mathbf{3}}, \mathbf{1})$. In this case our factorization is

$P_{(\overline{3}, 1)}=P_{u^{c}} P_{d^{c}}=0$,

where each factor is again displayed in Table 6. Also omitting $\lambda_{1}=D_{a}=0$, we have

$S \cdot E_{2 D, u^{c}}=1: \quad e_{2}=b_{3} b_{6} e_{0}+b_{5} e x=X=0$,

$S \cdot E_{2 D, d^{c}}=0: \quad e_{2}=b_{3} b_{6} e_{0}+b_{5} e x=X=Y_{1}=0$,

$S \cdot E_{2 F, u^{c}}=0: \quad e_{2}=b_{3} b_{5} b_{6} e_{0}^{2} e_{1}+b_{5}\left(b_{2} b_{3}-b_{0} b_{5}\right) e e_{0} e_{1} x$

$+b_{3}^{2} e\left(b_{5} y+e e_{1} x^{2}\right)=X=Y_{1}=0$,

$S \cdot E_{2 F, d^{c}}=1: \quad e_{2}=b_{3} b_{5} b_{6} e_{0}^{2} e_{1}+b_{5}\left(b_{2} b_{3}-b_{0} b_{5}\right) e e_{0} e_{1} x$

$+b_{3}^{2} e\left(b_{5} y+e e_{1} x^{2}\right)=X=0$.

While the constraint $P_{u^{c}}=a_{1}^{2} b_{3}+a_{0}^{2} b_{5}=0$ makes the conditions in (79) automatically solve the equation $Y_{1}=0$, this is not in the case for the $d^{c}$ curve in (80). The same situation holds for $E_{2 F}$. The rest of the intersection is the same as in the previous case, $S \cdot E_{0}=-1, S \cdot E_{1}=0$.

There is still no matter curve for $(\mathbf{1}, \mathbf{2})$ for the factorization (58); For generic $a_{i}$ and $d_{i}$, we cannot solve the six parameters in (49).

Matter curve for $(1,2)$ With the factorization at $X=0$, one of $E_{G}$ and $E_{H}$ has a solution in the form

$$
\begin{aligned}
E_{G}: p+q x+r y & =\left(a_{0} e_{0} z+a_{1} t\right)\left(g_{0} e_{0}^{2} z^{2}\right. \\
\left.+g_{1} e_{0} z t+g_{2} t^{2}\right) & =0,
\end{aligned}
$$

with the constraint $a_{0} g_{1}+a_{1} g_{0}=0$. This is regarded as the definition of $E_{G}$ from now on, and the other part, $E_{H}$, is left untouched. Thus the modified defining equation of $E_{G}$ contains the factor $Y_{1}=0$, and the condition is redundant. This is the reason why we have no further factorization of $P_{(2,1)}$. Therefore we have the intersection structure

$S \cdot E_{G}=1: \quad P_{(2,1)}=X=Y_{1}=\lambda_{1}=D_{a}=0$,

$S \cdot E_{H}=0$.

At the same time we can distinguish the lepton doublet from the down-type Higgs doublet by extra $U(1)$ quantum numbers, different from the hypercharge.

Matter curve for $(1,1)$ After the factorization to obtain the hypercharge symmetry $U(1)_{Y}$, we have a new charged singlet $e^{c}:(\mathbf{1}, \mathbf{1})_{1}$ under the SM group $S U(3) \times S U(2)_{L} \times U(1)_{Y}$, shown in Table 6. On the matter curve $P_{e^{c}}=0$, we have gauge symmetry enhancement $U(1)_{Y} \rightarrow S U(2)_{R}$, so that the resulting $S U(3) \times S U(2)_{L} \times S U(2)_{R}$ is still a subgroup of $S O(10)[56,57]$.
We note that $P_{e^{c}}=0$ is contained in the complete intersection between $Y_{1}$ and $Y_{2}$. Around here, the fiber equation (65) locally has the binomial structure of a deformed Kodaira $\mathrm{I}_{2}$ equation $x y=z_{1} z_{2}$, which describes nothing but this $S U(2)_{R}$ gauge symmetry $[26,27,33,34]$. Therefore the small resolution gives the $\mathbb{P}^{1}$ fiber (66) over the locus $P_{e^{c}}=0$, which we now call $S^{\prime}$. This $S^{\prime}$ will be related to the weight vector for $e^{c}$. Away from the intersection $P_{e^{c}}=0$ in the base $B$, its fiber described by (66) is already a $\mathbb{P}^{1}$, which we call $E^{\prime}$. These two have the McKay correspondence of the affine $S U(2)_{R}$, namely $S^{\prime} \cdot E^{\prime}=2$. Furthermore, we can show that $S$ intersects the entire fiber $S^{\prime}+E^{\prime}$ at a single point [33]. Therefore $S^{\prime}$ provides the desired intersection, giving the correct hypercharge of $e^{c}$,

$S \cdot S^{\prime}=-1$.

\subsection{Hypercharge generator from the embedding}

In the previous section, we have studied the intersections of the new divisor $S$ in (68) with various divisors, or, to be more precise, the intersection numbers between their $\mathbb{P}^{1}$ fibers in the sense of (31). However, on various loci $P_{f}=0$ each of the divisors $E_{1}, E_{2}, E$ may further degenerate into many, and we can recollect the results in terms of the intersections among $E_{1}, E_{2}, E$, and $S$. For example, the relation (78) may be recollected as $S \cdot E_{2}=1$, since this relation is independent of any specific locus $D_{a}=D_{b}=0$ on which we calculate.

Therefore we summarize our result as follows. We have a McKay correspondence of intersections of the $\mathbb{P}^{1}$ fibers,

$$
\begin{aligned}
& \begin{array}{llll}
E_{1} & E_{2} & S & E
\end{array} \\
& \begin{array}{l}
E_{1} \\
E_{2} \\
S \\
E
\end{array}\left(\begin{array}{cccc}
-2 & 1 & 0 & 0 \\
1 & -2 & 1 & 0 \\
0 & 1 & -2 & 1 \\
0 & 0 & 1 & -2
\end{array}\right)=-A_{S U(5)}
\end{aligned}
$$

being the negative of the Cartan matrix of $S U$ (5). The divisor $S$ provides the 'fourth root' of $S U(5)$. This fact is welcome, since the hypercharges are correctly given to the fields when we choose $S$ as the generator with a suitable normalization. The divisors $E_{1}, E_{2}, E$ may be blown down to zero size to recover the non-Abelian singularity $S U(3) \times S U(2)$. However, the divisor $S$ cannot be blown down while maintaining the factorization of Eq. (64), since we cannot allow for a conifold singularity with higher codimension. Therefore, at best we can have the gauge group $S U(3) \times S U(2) \times U(1)$, not the full $S U(5)$.

However, the Poincaré dual two-form to the divisor $S$ is not exactly what we want as the hypercharge generator, since we need a disconnected $\omega_{Y} \simeq S$ from the other group $S U(3) \times$ $S U(2)$ as 


$$
\int_{\hat{Y}} w_{Y} \wedge E_{i} \wedge D_{a} \wedge D_{b}=0 \text { for } E_{1}, E_{2}, E .
$$

We may form a linear combination of $S$ with the $E_{i}$ to have the desired property. This means to find the coefficients $t_{i}$ in (71) in the Shioda map [52-55] as done using the following mnemonics. First, take the inverse Cartan matrix $A^{-1}$ of the enhanced gauge symmetry of rank $r+1$. The Dynkin basis is defined to provide a convenient orthogonal relation between the roots $\alpha_{i}$ and the weights $w^{i}$ in a group under consideration,

$\alpha_{i} \cdot w^{j}=\delta_{i}^{j}, \quad a_{i}=\sum_{j} A_{i j} w^{j}$,

where the Cartan matrix provides the product metric and the sum is done over all the weights in that algebra. The symmetry breaking is described by deleting the $j$ th node of the Dynkin diagram and the resulting unbroken symmetry with the Cartan matrix being the one without the $j$ th row and the $j$ th column. From the orthonormality relation, what we need here is to take the $j$ th row of the inverted Cartan matrix as the coefficients $t_{i}$ of linear combinations of root divisors $E_{i}$. In our case,

$A_{S U(5)}^{-1}=\frac{1}{5}\left(\begin{array}{cccc}4 & 3 & 2 & 1 \\ 3 & 6 & 4 & 2 \\ 2 & 4 & 6 & 3 \\ 1 & 2 & 3 & 4\end{array}\right)$.

The symmetry breaking $S U(5) \rightarrow S U(3) \times S U(2) \times U(1)$ is done by removing the third row (and removing the extended root of the $S U(5))$. Therefore, we take the third row $(2,4,6,3)$, neglecting the overall normalization $1 / 5$, to obtain 2, 4, 6, 3 for the coefficients of $E_{1}, E_{2}, S$, and $E$, respectively. This always guarantees the integral charges under this $U(1)$. We finally have the hypercharge generator

$w_{Y}=-\left[S-Z-\overline{\mathcal{K}}-a_{1}+\frac{1}{6}\left(2 E_{1}+4 E_{2}+3 E\right)\right]$,

with the overall normalization chosen according to the conventional charge. Applying this to any component $E_{f}$ of each field $f$ gives the hypercharge $Y_{f}=\int_{E_{f}} w_{Y}$ as

$Y_{q}=\frac{1}{6}, Y_{X}=-\frac{5}{6}, Y_{u^{c}}=-\frac{2}{3}, Y_{d^{c}}=\frac{1}{3}, Y_{l}=-\frac{1}{2}, Y_{e^{c}}=1$.

It is highly non-trivial that every component has a different inner product with $6 S$ and $2 E_{1}+4 E_{2}+3 E$, but their sum is always the same, as it should be for the components in the same multiplet.

\subsection{Further factorization}

The model we have been building so far cannot be realistic for several reasons, as given below.

1. The spectrum so far, listed in Table 6, cannot take into account the electroweak Higgs fields.

2. To have a four-dimensional chiral spectrum, we have to turn on $G$-flux. If we turn on a universal $G$-flux along the entire ' $S U(5)_{\perp}$ ' $\operatorname{part}^{3}$ we have a partial unification relation of its commutant in $E_{8}$, namely $S U(5)$. That is, the SM fields belonging to the same representation of $S U(5)$ have the same number of generations among themselves. For example, we have $n_{q}=n_{u^{c}}=n_{e^{c}}$ from the 10 relation of the $S U$ (5) unification, where $n_{f}$ is the number of generations of a matter field $f$. We observe the same number of generations for the fields belonging to $\overline{5}$ as well. Therefore, we expect a stronger relation from a larger unification group, for instance of $S O(10)$.

These problems are best solved as follows, if we further factorize $\left.\hat{P}\right|_{X=0}$.

1. It is well known that the electroweak Higgs has the same quantum number as the lepton doublet under the SM group. By further factorization, the matter curve $P_{l_{\circ}}$ for $(2,1)$ further degenerates into those of the lepton doublet and the Higgs doublet. They are distinguished by different localization and/or different quantum numbers under the other unbroken group.

2. For a stronger unification relation, we may turn on the $G$-flux along a part smaller than $S U(5)_{\perp}$, for instance $S U(4)_{\perp}$, which gives a larger commutant for the unification relation, for instance of $S O(10)$. This procedure is only possible when we have the $S U(4)_{\perp}$ or a subgroup thereof, obtainable by further factorization.

Note that always the unbroken group is the SM group $S U(3) \times S U(2) \times U(1)_{Y}$, purely determined by the type of singularity (58), regardless of the choice of $G$-flux. The former determines the field content (quantum numbers) in the low-energy range, whereas the latter gives the unification relations for the number of generations. In the spectral cover construction, it was shown that factorization with the spectral cover $S[U(3) \times U(1) \times U(1) \times U(1)]$ is most realistic [41], and that the same applies to our F-theory version.

\footnotetext{
${ }^{3}$ For convenience we call this $S U(5) \perp$ part the commutant group of the $S U(3) \times S U(2) \times U(1)$ in $E_{8}$, just borrowing the nomenclature of the spectral cover construction. The other commutant in this case is the hypercharge $U(1)_{Y}$ since the abelian group commutes with itself.
} 
Therefore we further factorize the fiber equation at $X=0$ in the form (we will drop the factor $e_{0}$ and $z$ for simplicity) ${ }^{4}$ by tuning the parameters:

$$
\begin{aligned}
\left.\hat{P}\right|_{X=0}= & \left(a_{0}+a_{1} t\right)\left(b_{0}+b_{1} t\right)\left(d_{0}+d_{1} t\right)\left(f_{0}+f_{1} t\right. \\
& \left.+f_{2} t^{2}+f_{3} t^{3}\right) \equiv Y_{1} Y_{2} Y_{3} Y_{4}
\end{aligned}
$$

with the constraint

$$
a_{1} b_{0} d_{0} f_{0}+a_{0} b_{1} d_{0} f_{0}+a_{0} b_{0} d_{1} f_{0}+a_{0} b_{0} d_{0} f_{1}=0 \text {. }
$$

We may rewrite the resulting fiber equation as

$$
X Q=Y_{1} Y_{2} Y_{3} Y_{4}
$$

by introducing a holomorphic polynomial $Q$ in $z$ and $t$. We have six conifold singularities $X=Q=Y_{i}=Y_{j}=0$ with $(i, j)=(1,2),(1,3),(1,4),(2,3),(2,4),(3,4)$. Regarding each variable as coordinate in $\mathbb{C}^{6}$, the conifold singularities are viewed as surfaces meeting at six lines $X=$ $Q=Y_{i}=Y_{j}=Y_{k}=0$ and in turn the latter meet at one point $X=Q=Y_{1}=Y_{2}=Y_{3}=Y_{4}$, forming a bouquet. Counting the number of factors $Y_{i}$, we can easily show that we need small resolutions at three places. As a result, we have as many new $U(1)$. So we can extend the ambient space by introducing $\left(\mathbb{P}^{1}\right)^{3}$ with homogeneous coordinates $\left(\lambda_{1}, \lambda_{2}\right),\left(\mu_{1}, \mu_{2}\right),\left(v_{1}, v_{2}\right)$, and performing small resolutions:

$$
\begin{aligned}
& Y_{1} \lambda_{2}=Q \lambda_{1}, \quad Y_{2} \mu_{2}=\mu_{1} v_{2}, \\
& Y_{3} \lambda_{1} \mu_{1} v_{1}=\lambda_{2} \mu_{2}, \quad Y_{4} v_{2}=X v_{1} .
\end{aligned}
$$

These equations define four hypersurfaces in the extended ambient space, and our new resolved Calabi-Yau manifold $\hat{Y}$ is the complete intersection thereof. The resulting manifold is smooth since the collections of four gradients on all the hypersurfaces are linearly independent. We have other possible resolutions if we take different permutations of the $Y_{i}$, and they are related by flop transitions [34].

Consequently, we have new exceptional hypersurfaces containing the sections $X=Y_{i}=0$ for $i=1,2,3$ :

$$
\begin{aligned}
& S: \quad \lambda_{1}=\mu_{2}=v_{2}=0 \Longrightarrow X=Y_{1}=0, \\
& S_{X}: \quad \lambda_{2}=\mu_{1}=v_{1}=0 \Longrightarrow X=Y_{2}=Q=0, \\
& S_{Z}: \quad \lambda_{2}=\mu_{2}=v_{2}=0 \Longrightarrow X=Y_{3}=Q=0 .
\end{aligned}
$$

The two extra $U$ (1) charges we call $X$ and $Z$. This $S$ divisor has essentially the same definition as that in the previous factorization of (68), having the same group and Lorentz properties. As before, the newly found divisors $S_{X}$ and $S_{Z}$ provide a 'missing' Cartan subalgebra of $S U(5)$ or $S O(10)$, respectively. We can recycle the $U(1)$ generators $w_{Y}$, since the latter satisfies all of the requirements of the Cartan subalgebra (88)

\footnotetext{
${ }^{4}$ In what follows we have new definitions of the $Y_{i}$ and $Q$, etc., and they are not related to similar definitions given in the previous section.
}

and the whole set of generators satisfy the desirable conditions (69) and (70). Thus we determine the new generators with normalization,

$$
\begin{aligned}
w_{X}= & 5\left(S_{X}-Z-\overline{\mathcal{K}}-b_{1}\right)+2 E_{1}+4 E_{2}+6 E+3\left(-6 w_{Y}\right) \\
w_{Z}= & 4\left(S_{Z}-Z-\overline{\mathcal{K}}-d_{1}\right)+2 E_{1}+4 E_{2}+6 E \\
& +5 w_{X}+3\left(-6 w_{Y}\right)
\end{aligned}
$$

where the factor -6 in front of $w_{Y}$ is due to the special fractional convention of the hypercharge. Since $S_{X}$ and $S_{Z}$ are going to belong to $S O(10)$ and $E_{6}$, respectively, the coefficients are also found from the inverted Cartan matrices,

$$
\begin{gathered}
A_{S O(10)}^{-1}=\frac{1}{4}\left(\begin{array}{ccccc}
4 & 4 & 4 & 2 & 2 \\
4 & 8 & 8 & 4 & 4 \\
4 & 8 & 12 & 6 & 6 \\
2 & 4 & 6 & 5 & 3 \\
2 & 4 & 6 & 3 & 5
\end{array}\right), \\
A_{E_{6}}^{-1}=\frac{1}{3}\left(\begin{array}{cccccc}
4 & 5 & 6 & 4 & 2 & 3 \\
5 & 10 & 12 & 8 & 4 & 6 \\
6 & 12 & 18 & 12 & 6 & 9 \\
4 & 8 & 12 & 10 & 5 & 6 \\
2 & 4 & 6 & 5 & 4 & 3 \\
3 & 6 & 9 & 6 & 3 & 6
\end{array}\right) .
\end{gathered}
$$

The further generalization is straightforward. The spectrum of the fields and the corresponding charges are shown in Table 1 in Ref. [41].

\section{Comment on gauge coupling unification}

With localization of each gauge theory on a complex surface $S_{4}$ in $B$, a part of an eight-dimensional worldvolume, we have the following field theory limit having dimensional reduction [8-10]:

$$
-\frac{e^{-\phi}}{(2 \pi \alpha \prime)^{4}} \int_{S_{4} \times \mathbb{R}^{4}} \mathrm{~d}^{8} x F_{8 D}^{2}=-\frac{\mathrm{Vol} S_{4}}{4 g_{\mathrm{YM}}^{2}} \int_{\mathbb{R}^{4}} \mathrm{~d}^{4} x F_{4 D}^{2}+\cdots,
$$

with the vacuum expectation value of the dilaton $e^{\phi}$ becoming the string coupling. In the IIB string theory limit, this $\mathrm{Vol} S_{4}$ is interpreted as the effective volume of the cycle wrapped by dynamical seven-branes with both NSNS and RR charges.

The volumes of $S_{(3)}$ and $S_{(2)}$, respectively, spanned by the $S U$ (3) locus $W \equiv E_{0}: e_{0}=0$ (in $B$ ) and the $S U$ (2) locus $W^{\prime}: w^{\prime}=0$ are related, using (87),

$\operatorname{Vol}_{(3)}=\frac{1}{2} \int_{S_{(3)}} J \wedge J=\frac{1}{2} \int_{B} W \wedge J \wedge J$ 


$$
\begin{aligned}
& =-\frac{1}{2} \int_{\hat{Y}} E_{2} \wedge S \wedge J \wedge J \\
& =-\frac{1}{2} \int_{\hat{Y}} E \wedge S \wedge J \wedge J=\frac{1}{2} \int_{B} W^{\prime} \wedge J \wedge J \\
& =\frac{1}{2} \int_{S_{(2)}} J \wedge J=\operatorname{Vol} S_{(2)},
\end{aligned}
$$

where $J$ is the Kähler form of $\hat{Y}$. Therefore we have the same worldvolume for these two non-Abelian gauge groups. Here the calibrated geometry plays a role: the effective volumes are given by the intersection numbers, independent of the scaling factors of the coordinates.

The gauge coupling of the hypercharge $U(1)$ can readily be determined in the relation to the enhanced group such as $S U$ (5). It should be a global limit $b_{6}=0$, i.e. not local gauge symmetry enhancement on the matter curves,

$$
\begin{aligned}
\left.\hat{P}\right|_{b_{6}=0}= & e_{1}^{2} e_{2}^{3}\left[x^{3} e^{3} e_{1}-y^{2} e^{2} e_{2}+b_{5} e^{2} x y z\right. \\
& +b_{4} e_{0} e_{1} e^{2} x^{2} z^{2}+b_{3} e_{0}^{2} e_{1} e_{2} e y z^{3} \\
& \left.+b_{2} e_{0}^{3} e_{1}^{2} e_{2} e x z^{4}+b_{0} e_{0}^{5} e_{1}^{3} e_{2}^{2} z^{6}\right],
\end{aligned}
$$

with the tuned parameters $b_{i}$ in (58). We are relieved to see that in this limit, the two-cycles $e_{0}, e_{1}, e_{2}$ are identical to those in the standard resolution of the $S U(5)$ singularity $\mathrm{I}_{5}$ (see, e.g. [33], after renaming $e_{2} \rightarrow e_{4}$ ). The worldvolume is provided by the divisor $W_{0}: e_{0}=\left.\hat{P}\right|_{b_{6}=0}=0$, which is the same as $W$ of the $S U(3)$. Thus

$$
\begin{aligned}
\operatorname{Vol} S_{(5)}= & \frac{1}{2} \int_{S_{(5)}} J \wedge J=\frac{1}{2} \int_{B} W_{0} \wedge J \wedge J \\
& =\frac{1}{2} \int_{B} W \wedge J \wedge J=\operatorname{Vol} S_{(3)} .
\end{aligned}
$$

The fact that $W, W^{\prime}, W_{0}$ have the same volume is obvious since the $S U(3)$ and the $S U(2)$ are obtained by deforming the $S U$ (5) singularity. It is not affected by another deformation arising from the resolution $S$ of the conifold singularity. The volume of the $\mathbb{P}^{1}$ fiber of $S$ can never be zero so there cannot be unbroken $S U(5)$. Nevertheless the gauge couplings are unaffected by the volume of this $\mathbb{P}^{1}$, and in the low-energy limit we just have heavy $X, Y$ gauge multiplets.

In this limit, $S$ provides the Cartan subalgebra element related to the hypercharge, as seen in the relation (87); thus

$$
-\frac{1}{4 g^{2}} \operatorname{tr} F_{S U(5)}^{2}=-\frac{1}{4 g^{2}}\left(\operatorname{tr} F_{S U(3)}^{2}+\operatorname{tr} F_{S U(2)}^{2}+\operatorname{tr} F_{U(1)}^{2}\right),
$$

where we defined the gauge field as matrix valued, $A_{M}=$ $A_{M}^{a} t^{a}, \operatorname{tr} t^{a} t^{b}=\frac{1}{2} \delta^{a b}$. In particular, from the Cartan matrix (87), the generator $S$ is related to the Cartan element with $t=\frac{1}{\sqrt{60}} \operatorname{diag}(2,2,2,-3,-3)$. The two-cycle $w_{Y}$ is just a modification of that of $S$, and the linear transformation within the same group $S U$ (5) (otherwise even the definition (89) does not make sense) is just a transformation that does not affect the gauge coupling. Thus the gauge coupling of the hypercharge $U(1)_{Y}$ should be related by group theory of the unified group $S U(5)$ embedding $S U(3) \times S U(2) \times U(1)_{Y}$, in the standard way. Normalizing the $U(1)$ charge of the $e^{\mathrm{c}}$ to be 1 , we fix the coupling as

$g^{2}=g_{3}^{2}=g_{2}^{2}=\frac{3}{5} g_{Y}^{2}$,

with the Weinberg mixing angle at this string theory scale

$\sin \theta_{W}^{0}=\frac{g_{Y}^{2}}{g_{2}^{2}+g_{Y}^{2}}=\frac{3}{8}$

consistent with the observation. For any $U(1)$ being embedded in a certain GUT group, we may use this method. However, it is an open question whether every $U(1)$ obtainable in F-theory has such an embedding.

As regards this coupling relation, we have a threshold correction to the $U(1)$ gauge couplings, if there is a non-trivial $G$-flux along these $U(1)$ directions [11,58,59]. When we construct the SM group at the string scale, we have an option not to turn on $G$-flux along the hypercharge direction even if we want it to have gauge symmetry. For GUT such as $S U(5)$, we may break it by turning on $G$-flux without breaking gauged hypercharge symmetry $[8,9,11]$. For other $U(1)$ symmetries constructed for the realistic model building, it is desirable for them to be broken down by $G$-flux. Then via the Stückelberg mechanism, the corresponding gauge boson acquires mass and the symmetry becomes global.

\section{Conclusions}

We analyzed the standard model gauge group $S U(3) \times$ $S U(2) \times U(1)$, and its accompanying matter fields, constructed in F-theory. The non-Abelian part $S U(3) \times S U(2)$ is described by a surface singularity of Kodaira type, which locally looks like $\mathrm{I}_{3}$ and $\mathrm{I}_{2}$, which can be regarded as a nongeneric deformation of the $S U(5)$ singularity $\mathrm{I}_{5}$. Each factor group is, respectively, supported by different divisors $w=0$ and $w^{\prime}=0$, which are related by the coordinate transformation given in (19). Nevertheless they are described by a single equation (20). The resolution analysis revealed that the SM group should be the rank three group along the $E_{n}$ series, distinguished from the naïve product of $S U(3)$ and $S U(2)$ in the following sense. The well-known reason is that the desired matter curves of the SM are obtained in this singularity with particular gauge symmetry enhancements. Now the blow-up analysis shows that the exceptional divisors from different simple groups mix in some particular way on the matter curves, yielding matter fields with the desired charges. This fact is due to the fact that the $S U$ (3) and the $S U$ (2) group 
components are connected by coordinate transformations and the blow up cannot be done independently.

The Abelian part $U(1)$ is obtained by the 'factorization method' making use of an extra section in the elliptic fiber of an internal manifold. Restricting the elliptic fiber equation on a special divisor, we can determine the relation between the elliptic fiber equation and a spectral cover equation. For the latter, we know that the factorization of the equation is related to the gauge symmetry enhancement in certain group directions. The resolution at the conifold singularity originating from this factorization gives rise to the two-form harboring the desired gauge group, having the correct assignment of $U(1)$ charges. This new two-form and the corresponding divisor should be understood in terms of a certain unified group, and from this the conventional $S U(5)$ gauge coupling unification relation is achieved if no flux is turned on in the $U$ (1) part.

We may apply this method to a direct construction of the Standard Model in the native F-theory context. On top of the desired hypercharge $U(1)$ obtained by factorizing the spectral cover, with further factorization done in Sect. 3.4, we are able to successfully reproduce the observed three generations of quarks and leptons [24,25]. There are some hurdles to overcome from further phenomenological requirements. For instance, we may also obtain one pair of Higgs doublets as in the minimal supersymmetric standard model, by turning on non-universal flux [41], but in this case we need further tuning to achieve decoupling of a colored Higgs. Mathematically, on the other hand, the appearance of an extra $U(1)$ as an element of the Cartan subalgebra in a larger unified group is very suggestive, so it would be interesting to extend this work to determine an additional systematic method for groups involving multiple $U(1)$.

Acknowledgments The author is grateful to Ralph Blumenhagen, Thomas Grimm, Stefan Groot-Nibbelink, Hirotaka Hayashi, Seung-Joo Lee, Christopher Mayrhofer, and Timo Weigand for discussions and correspondences. This work is partly supported by the National Research Foundation of Korea with Grant Number 2012-R1A1A1040695.

Open Access This article is distributed under the terms of the Creative Commons Attribution License which permits any use, distribution, and reproduction in any medium, provided the original author(s) and the source are credited.

Funded by $\mathrm{SCOAP}^{3}$ / License Version CC BY 4.0.

\section{References}

1. H. Georgi, S.L. Glashow, Phys. Rev. Lett. 32, 438-441 (1974)

2. J.C. Pati, A. Salam, Phys. Rev. D 10, 275-289 (1974)

3. P. Ramond, Introduction to Exceptional Lie Groups and Algebras, preprint CALT-68-577

4. C. Vafa, Nucl. Phys. B 469, 403 (1996). arXiv:hep-th/9602022

5. D.R. Morrison, C. Vafa, Nucl. Phys. B 476, 437 (1996). arXiv:hep-th/9603161
6. D.R. Morrison, C. Vafa, Nucl. Phys. B 473, 74 (1996). arXiv:hep-th/9602114

7. M. Bershadsky, A. Johansen, T. Pantev, V. Sadov, Nucl. Phys. B 505, 165 (1997). arXiv:hep-th/9701165

8. C. Beasley, J.J. Heckman, C. Vafa, JHEP 0901, 058 (2009). arXiv:0802.3391 [hep-th]

9. C. Beasley, J.J. Heckman, C. Vafa, JHEP 0901, 059 (2009). arXiv:0806.0102 [hep-th]

10. R. Donagi, M. Wijnholt, Adv. Theor. Math. Phys. 15, 1237 (2011). arXiv:0802.2969 [hep-th]

11. R. Donagi, M. Wijnholt, Adv. Theor. Math. Phys. 15, 1523 (2011). arXiv:0808.2223 [hep-th]

12. R. Tatar, T. Watari, Nucl. Phys. B 747, 212 (2006). hep-th/0602238

13. H. Hayashi, R. Tatar, Y. Toda, T. Watari, M. Yamazaki, Nucl. Phys. B 806, 224 (2009). arXiv:0805.1057 [hep-th]

14. H. Hayashi, T. Kawano, R. Tatar, T. Watari, Nucl. Phys. B 823, 47 (2009). arXiv:0901.4941 [hep-th]

15. R. Donagi, M. Wijnholt, Commun. Math. Phys. 326, 287 (2014). arXiv:0904.1218 [hep-th]

16. K.-S. Choi, T. Kobayashi, R. Maruyama, M. Murata, Y. Nakai, H. Ohki, M. Sakai, Eur. Phys. J. C 67, 273 (2010). arXiv:0908.0395 [hep-ph]

17. J.J. Heckman, A. Tavanfar, C. Vafa, JHEP 1008, 040 (2010). arXiv:0906.0581 [hep-th]

18. E. Palti, JHEP 1207, 065 (2012). arXiv:1203.4490 [hep-th]

19. A.P. Braun, T. Watari, JHEP 1307, 031 (2013). arXiv:1301.5814 [hep-th]

20. M. Esole, R. Savelli, JHEP 1306, 027 (2013). arXiv:1209.1633 [hep-th]

21. S. Katz, D.R. Morrison, S. Schafer-Nameki, J. Sully, JHEP 1108, 094 (2011)

22. M. Bershadsky, K.A. Intriligator, S. Kachru, D.R. Morrison, V. Sadov, C. Vafa, Nucl. Phys. B 481, 215-252 (1996)

23. S.H. Katz, C. Vafa, Nucl. Phys. B 497, 146 (1997). arXiv:hep-th/9606086

24. K.-S. Choi, T. Kobayashi, Phys. Lett. B 693, 330 (2010). arXiv:1003.2126 [hep-th]

25. K.-S. Choi, Nucl. Phys. B842, 1-32 (2011). arXiv:1007.3843 [hepth]

26. T.W. Grimm, T. Weigand, Phys. Rev. D 82, 086009 (2010). arXiv:1006.0226 [hep-th]

27. S. Krause, C. Mayrhofer, T. Weigand, JHEP 1208, 119 (2012). arXiv:1202.3138 [hep-th]

28. J. Marsano, N. Saulina, S. Schafer-Nameki, arXiv:1107.1718 [hepth]

29. M.J. Dolan, J. Marsano, N. Saulina, S. Schafer-Nameki, Phys. Rev. D 84, 066008 (2011). arXiv:1102.0290 [hep-th]

30. S. Krause, C. Mayrhofer, T. Weigand, Nucl. Phys. B 858, 1 (2012). arXiv:1109.3454 [hep-th]

31. R. Friedman, J. Morgan, E. Witten, Commun. Math. Phys. 187, 679 (1997). hep-th/9701162

32. K.-S. Choi, H. Hayashi, JHEP 1206, 009 (2012). arXiv: 1203.3812 [hep-th]

33. C. Mayrhofer, E. Palti, T. Weigand, JHEP 1303, 098 (2013). arXiv:1211.6742 [hep-th]

34. M. Esole, S.-T. Yau, arXiv:1107.0733 [hep-th]

35. B. Andreas, D. Hernandez Ruiperez, Adv. Theor. Math. Phys. 9, 253 (2005). hep-th/0410170

36. V. Braun, T.W. Grimm, J. Keitel, JHEP 1309, 154 (2013). arXiv: 1302.1854 [hep-th]

37. M. Cvetic, D. Klevers, H. Piragua, JHEP 1306, 067 (2013). arXiv:1303.6970 [hep-th]

38. M. Cvetic, A. Grassi, D. Klevers, H. Piragua, arXiv:1306.3987 [hep-th]

39. V. Braun, T.W. Grimm, J. Keitel, arXiv:1306.0577 [hep-th] 
40. J. Borchmann, C. Mayrhofer, E. Palti, T. Weigand, Nucl. Phys. B 882, 1 (2014). arXiv:1307.2902 [hep-th]

41. K.-S. Choi, Phys. Lett. B 718, 218 (2012). arXiv:1109.5867 [hepth]

42. I. Antoniadis, G.K. Leontaris, Eur. Phys. J. C 73, 2670 (2013). arXiv:1308.1581 [hep-th]

43. J.C. Callaghan, S.F. King, G.K. Leontaris, JHEP 1312, 037 (2013). arXiv:1307.4593 [hep-ph]

44. J.C. Callaghan, S.F. King, JHEP 1304, 034 (2013). arXiv:1210.6913 [hep-ph]

45. G.K. Leontaris, PoS CORFU 2011, 095 (2011). arXiv:1203.6277 [hep-th]

46. H.P. Nilles, S. Ramos-Sanchez, P.K.S. Vaudrevange, AIP Conf. Proc. 1200, 226 (2010). arXiv:0909.3948 [hep-th]

47. J. Tate, Modular Functions of One Variable IV. Lecture Notes in Mathematics, vol. 476 (Springer, Berlin, 1975), pp. 33-52

48. The easiest way to see it is looking at the moment map or FayetIliopoulos constraint. See e.g. M. Blaszczyk, S. Groot Nibbelink, F. Ruehle, JHEP 1205, 053 (2012). arXiv:1111.5852 [hep-th]

49. T.W. Grimm, M. Kerstan, E. Palti, T. Weigand, JHEP 1112, 004 (2011). arXiv:1107.3842 [hep-th]

50. T.W. Grimm, H. Hayashi, JHEP 1203, 027 (2012). arXiv:1111.1232 [hep-th]
51. J. Marsano, N. Saulina, S. Schafer-Nameki, JHEP 0908, 046 (2009). arXiv:0906.4672 [hep-th]

52. T. Shioda, Mordell-Weil lattices and Galois representation. Proc. Jpn. Acad. A 65, 268 (1989)

53. T. Shioda, On the Mordell-Weil lattices. Comment. Math. Univ. St.Pauli 39, 211 (1990)

54. D.R. Morrison, D.S. Park, JHEP 1210, 128 (2012). arXiv:1208.2695 [hep-th]

55. M. Cvetic, T.W. Grimm, D. Klevers, JHEP 1302, 101 (2013). arXiv: 1210.6034 [hep-th]

56. K.-S. Choi, B. Kyae, Nucl. Phys. B 855, 1 (2012). arXiv: 1102.0591 [hep-th]

57. K.-S. Choi, J.E. Kim, Phys. Rev. D 83, 065016 (2011). arXiv:1012.0847 [hep-ph]

58. R. Blumenhagen, Phys. Rev. Lett. 102, 071601 (2009). arXiv:0812.0248 [hep-th]

59. J.J. Heckman, H. Verlinde, JHEP 1101, 044 (2011). arXiv:1005.3033 [hep-th] 